$\mathrm{gr}-\mathrm{qc} / 0005115$

HIP-2000-25/TH

June 21, 2000

\title{
Hawking Radiation from AdS Black Holes
}

\author{
Samuli Hemming \\ Esko Keski-Vakkurif \\ Helsinki Institute of Physics \\ P.O. Box 9 \\ FIN-00014 University of Helsinki \\ Finland
}

\begin{abstract}
We investigate Hawking radiation from black holes in $(\mathrm{d}+1)$-dimensional anti-de Sitter space. We focus on s-waves, make use of the geometrical optics approximation, and follow three approaches to analyze the radiation. First, we compute a Bogoliubov transformation between Kruskal and asymptotic coordinates and compare the different vacua. Second, following a method due to Kraus, Parikh, and Wilczek, we view Hawking radiation as a tunneling process across the horizon and compute the tunneling probability. This approach uses an anti-de Sitter version of a metric originally introduced by Painlevé for Schwarzschild black holes. From the tunneling probability one also finds a leading correction to the semi-classical emission rate arising from backreaction to the background geometry. Finally, we consider a spherically symmetric collapse geometry and the Bogoliubov transformation between the initial vacuum state and the vacuum of an asymptotic observer.
\end{abstract}

\footnotetext{
${ }^{1}$ E-mail: samuli.hemming@hip.fi

${ }^{2}$ E-mail: esko.keski-vakkuri@hip.fi
} 


\section{Introduction}

Anti-de Sitter black holes play a major role in the AdS/CFT conjecture [1] (see [2] for a review), and they have also received interest in the context of brane-world scenarios based on the setup of Randall and Sundrum [3, 4]. The purpose of this note is to briefly investigate the basic property of black holes, Hawking radiation, in anti-de Sitter spacetimes.

In the 2+1 dimensional special case, the BTZ black holes [5], Hawking radiation of a massles conformally coupled scalar field was investigated in detail by Hyun et. al. [6]. Here we will instead take a more generic approach (while sacrifying detail), and extend some standard ways to analyze Hawking radiation from Schwarzschild black holes to the anti-de Sitter case in a generic dimension. The standard methods in question are to investigate eternal black holes and the role of different vacua based on different boundary conditions at the horizons, and to investigate radiation in a spherical collapse geometry while the quantum field is in a natural initial vacuum. Customarily, the focus is on finding the leading thermal characteristics of the radiation. For this purpose it is sufficient to invoke a geometric optics approximation which neglects backscattering of outgoing waves from the spacetime curvature and essentially reduces the problem to a two dimensions. The neglected effects would give rise to a gray-body factor in the thermal emission spectrum. The gray-body factors can be found by investigating absorption by AdS black holes.

We will also investigate the back-reaction to the geometry by taking into account the selfinteraction effect analyzed by Kraus, Parikh and Wilczek [9, 10, 11] (see also [12]). This approach views Hawking radiation as a tunneling process across the horizon. Here we extend the approach of [9, 10, 11] to the anti-de Sitter case, and find similar results.

\section{Hawking radiation from eternal black holes}

One standard method to derive the Hawking radiation is to use a Bogoliubov transformation between two basis of annihilation and creation operators corresponding to mode expansions of the field operator in two preferred coordinate systems used to describe a black hole: the asymptotic coordinates and the Kruskal coordinates. In the asymptotic coordinates, the $\mathrm{AdS}_{d+1}$ black hole metric is (see e.g. [7])

$$
d s^{2}=-F(r) d t^{2}+\frac{d r^{2}}{F(r)}+r^{2} d \Omega_{d-1}^{2}
$$

where

$$
F(r)=1-\frac{\mu}{r^{d-2}}+r^{2}
$$


and we work in units where the AdS radius $l=1$. The parameter $\mu$ is proportional to the ADM mass $M$ of the black hole,

$$
M=\frac{(d-1) A_{d-1}}{16 \pi G_{d+1}} \mu
$$

where $G_{d+1}$ is Newton's constant in $d+1$ dimensions and $A_{d-1}=2 \pi^{d / 2} / \Gamma(d / 2)$ is the volume of a unit $(d-1)$-sphere. The explicit formula for the horizon radius $r_{H}$ can be found by solving the polynomial equation $F\left(r_{H}\right)=0$. For example, in $4+1$ dimensions,

$$
r_{H}=\sqrt{\frac{1}{2}(\sqrt{1+4 \mu}-1)} .
$$

Then, the Hawking temperature $T_{H}$ can be found by looking at the periodicity of the Euclidian section of the metric near $r_{H}$. The generic result is

$$
T_{H}=\frac{1}{4 \pi} F^{\prime}\left(r_{H}\right)
$$

where $^{\prime}=d / d r$. E.g. in $4+1$ dimensions,

$$
T_{H}=\frac{\sqrt{1+4 \mu}}{2 \pi r_{H}}
$$

To investigate mode solutions of a field equation, it is useful to introduce the tortoise coordinate $r_{*}$,

$$
r_{*}=\int_{r_{H}}^{r} \frac{d \hat{r}}{F(\hat{r})} .
$$

Again, in $4+1$ dimensions the explicit formula is

$$
r_{*}=\frac{1}{\sqrt{1+4 \mu}}\left(r_{0} \arctan \left(\frac{r}{r_{0}}\right)+\frac{1}{2} r_{H} \ln \left(\frac{r-r_{H}}{r+r_{H}}\right)\right),
$$

where $r_{H}$ is the horizon radius and $r_{0}$ is a shorthand notation denoting a radius

$$
r_{0}=\sqrt{\frac{1}{2}(\sqrt{1+4 \mu}+1)} .
$$

(In $4+1$ dimensions, the equation $F(r)=0$ has 4 complex zeroes: 2 real zeroes at $\pm r_{H}$ and 2 imaginary zeroes at $\pm i r_{0}$.) It is also convenient to introduce the null coordinates

$$
\begin{aligned}
& u=t-r_{*} \\
& v=t+r_{*}
\end{aligned}
$$


where $r_{*}$ is the tortoise coordinate. Then the metric takes the form

$$
d s^{2}=-F(r) d u d v+r^{2} d \Omega_{d-1}^{2}
$$

and the solutions to the wave equation are infalling and outgoing partial waves.

In the region outside the past and future horizons, the Kruskal coordinates $U, V$ are defined as follows:

$$
\begin{aligned}
U & =-\exp \left(-2 \pi T_{H} u\right) \\
V & =\exp \left(2 \pi T_{H} v\right)
\end{aligned}
$$

In $U, V$ coodinates, the metric takes the form

$$
d s^{2}=-\frac{F(r) e^{-4 \pi T_{H} r_{*}}}{\left(2 \pi T_{H}\right)^{2}} d U d V+r^{2} d \Omega_{d-1}^{2} .
$$

In $4+1$ dimensions, the explicit form is

$$
d s^{2}=-\frac{r_{H}^{2}}{1+4 \mu}\left(1+\frac{r_{0}^{2}}{r^{2}}\right)\left(r_{H}+r\right)^{2} \exp \left(-\frac{2 r_{0}}{r_{H}} \arctan \left(\frac{r}{r_{0}}\right)\right) d U d V+r^{2} d \Omega_{3}^{2} .
$$

In Kruskal coordinates, the metric can be extended over the whole spacetime, except for the origin $r=0$, which corresponds to the true curvature singularity of the black hole.

Next, we will focus on s-waves, adopt the geometric optics approximation, and truncate to two dimensions, following the classic paper by Unruh [8]. The discussion also overlaps with [13].

For a quantum field in the black hole background, there are two canonical choices for a natural vacuum state. If one wants to mimic a situation where the black hole is created by collapsing matter, one requires the field to be in a vacuum corresponding absence of positive energy modes in the $U$ and $v$ coordinates near the past horizon $V=0$. This boundary condition refers only to the past of the asymptotic region of spacetime, an is known as the Unruh vacuum. Another vacuum choice, the Hartle-Hawking vacuum, refers to a mixture of boundary conditions in the past and future horizons. Now one requires the absence of positive energy $U$ modes near $V=0$, and the absence of positive energy $V$ modes near $U=0$. Physically, this mimics a black hole in thermal equilibrium with an external heat bath. The task is to compare these two vacuua with the Boulware vacuum which is a natural vacuum for a fiducial observer in the asymptotic region. The Boulware vacuum corresponds to absence of positive energy $u$ and $v$ modes. To complete the discussion of vacua, we will also need to take into account the reflective boundary

\footnotetext{
${ }^{3}$ We thank D. Klemm for bringing this reference to our attention.
} 
condition at the boundary of the anti-de Sitter space. We will do at the end of this section.

To compute the Bogoliubov transformations between the different natural modes, we use the standard trick [8]. Let us focus on the outgoing modes first. To begin with, we define the modes

$$
\begin{aligned}
& \phi_{+, \omega}=\exp (-i \omega u)=\theta(-U)(-U)^{\frac{i \omega}{2 \pi T_{H}}}, \quad(U<0) \\
& \phi_{-, \omega}=\exp (i \omega u)=\theta(U) U^{-\frac{i \omega}{2 \pi T_{H}}}, \quad(U>0) .
\end{aligned}
$$

Then, to find a complete basis for positive energy $U$ modes we consider the linear combinations which extend over the whole $V=0$ line,

$$
\begin{aligned}
\phi_{1, \omega} & =\phi_{+, \omega}+C_{1} \phi_{-, \omega}^{*} \\
& =\theta(-U)(-U)^{\frac{i \omega}{2 \pi T_{H}}}+C_{1} \theta(U) U^{\frac{i \omega}{2 \pi T_{H}}} \\
\phi_{2, \omega} & =\phi_{-, \omega}+C_{2} \phi_{+, \omega}^{*} \\
& =\theta(U) U^{-\frac{i \omega}{2 \pi T_{H}}}+C_{2} \theta(-U)(-U)^{-\frac{i \omega}{2 \pi T_{H}}}
\end{aligned}
$$

We then demand that $\phi_{1, \omega}$ is a positive energy Kruskal mode: that for $\omega>0$ it must be analytic in the lower half complex $U$-plane. This condition is satisfied if the coefficient $C_{1}$ is

$$
C_{1}=\exp \left(-\frac{\omega}{2 T_{H}}\right)
$$

A similar condition for $\phi_{2, \omega}$ fixes the coefficient $C_{2}$ :

$$
C_{2}=\exp \left(-\frac{\omega}{2 T_{H}}\right)
$$

Hence $C_{1}=C_{2} \equiv C$.

From this we can compute the (unnormalized) $\alpha$ and $\beta$ Bogoliubov coefficients which denote the overlap of a positive energy $U$ mode with positive and negative energy $u$ modes:

$$
\begin{aligned}
& \alpha=\left(\phi_{1, \omega}, \phi_{+, \omega}\right)=\left(\phi_{2, \omega}, \phi_{-, \omega}\right) \propto 1 \\
& \beta=\left(\phi_{1, \omega}, \phi_{-, \omega}^{*}\right)=\left(\phi_{2, \omega}, \phi_{+, \omega}^{*}\right) \propto C
\end{aligned}
$$

(for $i=1,2$ ). The ratio of the two coefficients is thus

$$
\left|\frac{\beta}{\alpha}\right|^{2}=|C|^{2}=\exp \left(-\frac{\omega}{T_{H}}\right)
$$

Using the normalization condition of the Bogoliubov coefficients,

$$
|\alpha|^{2}-|\beta|^{2}=1
$$


we find that the average occupation number for positive energy $u$ modes, seen by a fiducial observer when the quantum field is in a vacuum with respect to positive energy $U$ modes, simplifies to the expected form of a Bose-Einstein distribution,

$$
\bar{n}_{\omega}=|\beta|^{2}=\frac{1}{\exp \left(\frac{\omega}{T_{H}}\right)-1}
$$

where $T_{H}$ is the Hawking temperature of the $\mathrm{AdS}_{d+1}$ black hole.

A similar relation holds between the $V$ and $v$ modes also. Now, we take into account the reflection from the boundary of adS space. Ref. [13] considered different possibilities for the boundary condition at infinity: Dirichlet, Neumann, and Robin boundary conditions. What is the preferred boundary condition? Let us leave the geometric optics approximation for the moment and consider mode solutions to the exact wave equation for a free scalar field in the adS-black hole space time. In Minkowski signature, the mode solutions can fall into two categories, nonnormalizable solutions $\phi_{\omega, \vec{k}}^{(-)}$and normalizable solutions $\phi_{\omega, \vec{k}}^{(+)}$, with the asymptotic behavior

$$
\phi_{\omega, \vec{k}}^{( \pm)}(t, r, \Omega) \rightarrow r^{-2 h_{ \pm}} \tilde{\phi}^{ \pm}(t, \Omega) \quad(r \rightarrow \infty),
$$

where $h_{ \pm}$are parameters related to the mass $\mu=$ and the dimension of the space $d$ by

$$
2 h_{ \pm}=\frac{1}{2}\left(d \pm \sqrt{d^{2}+4 \mu^{2}}\right) .
$$

The quantized field $\phi$ is expanded as a linear combination of the normalizable modes. Their decay behavior at the boundary corresponds to reflection. The exact mode solutions $\phi^{(+)}$are easy to find in $2+1$ dimensions in terms of hypergeometric functions [14, [15]. Near the black hole horizon, the normalizable modes reduce to a form

$$
\phi^{(+)} \sim\left(e^{-i \omega u}+e^{-i \omega v+i 2 \theta_{0}}\right) e^{-i n \phi}
$$

where $\theta_{0}$ is a phase shift factor, its exact form can be found in [14. Thus, in the geometric optics approximation, the modes which take into account the reflection from the boundary and are appropriate to a fiducial observer are a linear combination of the positive energy $u$ and $v$ modes,

$$
\phi_{\omega}=e^{-i \omega u}+e^{-i \omega v+i 2 \theta_{0}} .
$$

For Kruskal modes, one must consider the corresponding linear combination of the positive energy $U$ and $V$ modes. Thus, with the reflective boundary condition, the appropriate vacuum is the Hartle-Hawking vacuum. Then, a fiducial observer sees a thermal spectrum for both infalling and outgoing modes. In the eternal adS geometry, the Unruh vacuum 
is not well defined with respect to the boundary condition at infinity. It can be viewed as an artificial construction describing the very onset of radiation, where only outgoing modes are thermally excited and they have not yet reflected back from the We will return to this issue in Section 4.

\section{$3 \quad$ Hawking radiation as tunneling}

Recently, a method to describe Hawking radiation as a tunneling process, where a particle moves in dynamical geometry, was developed by Kraus and Wilczek [9, 10] and elaborated upon by Parikh and Wilczek [11. This method also gives a leading correction to the emission rate arising from loss of mass of the black hole correponding to the energy carried by the radiated quantum. This method was also investigated in the context of black holes in string theory [12], and it was demonstrated that in the string picture of microstates of the black hole, the correction to the emission rate corresponds to a difference between counting of states in the microcanonical and canonical ensembles. However, in all these investigations the black holes have had asymptotically flat spacetime geometry. We now extend the investigation to black holes in AdS spacetime. We will base our treatment on the presentation of [11].

A convenient trick in the method of [9, 10, 11] is to write the black hole metric in a coordinate system where constant time slices are flat, without a singularity at the horizon. In these coordinates, the Schwarzschild metric takes the form

$$
d s^{2}=-\left(1-\frac{2 M}{r}\right) d t^{2}+2 \sqrt{\frac{2 M}{r}} d t d r+d r^{2}+r^{2} d \Omega^{2} .
$$

These coordinates were first introduced 80 years ago by Painlevé [16], but then disappeared from general knowledge, until they were independently rediscovered in [9] and used to investigate black hole quantum mechanics. We will now derive an analogue of the Painlevé coordinates for AdS black holes, which we shall refer to as the AdS-Painlevé coordinates.

By analogue to the asymptotically flat black holes, the AdS-Painlevé coordinates should have the property that constant time slices of the AdS black hole metric (11) will have the same geometry as constant time slices of a global $\mathrm{AdS}_{d+1}$ metric

$$
d s^{2}=-\left(1+r^{2}\right) d t^{2}+\frac{d r^{2}}{\left(1+r^{2}\right)}+r^{2} d \Omega_{d-1}^{2} .
$$

Thus, we perform a coordinate transformation $t=\hat{t}+f(r)$ so the metric (1) takes the form

$$
d s^{2}=-F(r) d \hat{t}^{2}+2 f^{\prime}(r) F(r) d \hat{t} d r+\left(\frac{1}{F(r)}-F(r)\left(f^{\prime}(r)\right)^{2}\right) d r^{2}+r^{2} d \Omega^{2}
$$


and then demand that on constant $\hat{t}$ slices the metric reduces to

$$
d s^{2}=\left(1+r^{2}\right)^{-1} d r^{2}+r^{2} d \Omega^{2}
$$

This implies that

$$
f^{\prime}(r)=\frac{1}{r^{\frac{d-2}{2}} F(r)} \sqrt{\frac{\mu}{1+r^{2}}}
$$

so the AdS-Painlevé metric reads as follows:

$$
d s^{2}=-F(r) d \hat{t}^{2}+\frac{2}{r^{\frac{d-2}{2}}} \sqrt{\frac{\mu}{1+r^{2}}} d \hat{t} d r+\left(1+r^{2}\right)^{-1} d r^{2}+r^{2} d \Omega^{2} .
$$

Now we move on to discuss Hawking radiation. The (s-wave) quanta of a massless scalar field follow radial light-like geodesics

$$
\dot{r}=-\frac{\sqrt{\mu}}{r^{\frac{d-2}{2}}} \sqrt{1+r^{2}} \pm\left(1+r^{2}\right)
$$

where the $(+)$ sign corresponds to an outgoing geodesic and the $(-)$ sign corresponds to an ingoing geodesic, respectively. Next we take into account the response of the background geometry to an emitted quantum of frequency $\omega$. We keep the total mass $M$ of the spacetime fixed, but in order to take into account the energy carried by the quantum, we replace $\mu$ in (36) by $\mu^{\prime}$,

$$
\mu^{\prime} \equiv \frac{16 \pi}{(d-1) A_{d-1}}(M-\omega)
$$

Note that at the horizon,

$$
\left.\dot{r}\right|_{r_{H}}=0 \text {. }
$$

As the particle travels across the horizon from $r_{\text {in }}$ to $r_{\text {out }}$, its actionf receives an imaginary contribution

$$
\operatorname{Im} S=\operatorname{Im} \int_{r_{\text {in }}}^{r_{\text {out }}} p_{r} d r=\operatorname{Im} \int_{r_{\text {in }}}^{r_{\text {out }}} \int_{H} \frac{d H}{\dot{r}} d r
$$

where on the last line we switched the order of integration and used the Hamilton's equation $\dot{r}=\frac{d H}{d p_{r}}$. Next, we substitute from (36) the radial velocity along the outgoing geodesic, and use $d H=d(M-\omega)=-d \omega$ :

$$
\operatorname{Im} S=-\operatorname{Im} \int_{0}^{\omega} d \omega^{\prime} \int_{r_{\text {in }}}^{r_{\text {out }}} d r \frac{1+\sqrt{\frac{\mu^{\prime}}{r^{d-2}}\left(1+r^{2}\right)^{-1}}}{F(r)} .
$$

\footnotetext{
${ }^{4}$ Note that in the local point particle description used in this section, the issue of boundary conditions at infinity does not arise.
} 
The only imaginary contribution to the radial integral comes from the pole at $r_{H}$. Then

$$
\operatorname{Im} S=\pi \int_{0}^{\omega} d \omega^{\prime} \frac{2 r_{H}}{r_{H}^{2} d+(d-2)} .
$$

On the other hand, after solving for $r_{H}$ as a function of $\mu$, we can derive that

$$
r_{H}^{d-2} \frac{d r_{H}}{d \mu}=\frac{r_{H}}{r_{H}^{2} d+(d-2)} .
$$

Substituting this into the integral yields

$$
\begin{aligned}
\operatorname{Im} S & =\pi \frac{(d-1) A_{d-1}}{16 \pi} \int_{\mu-\frac{16 \pi}{(d-1) A_{d-1}} \omega}^{\mu} d \mu^{\prime} 2 r_{H}^{d-2} \frac{d r_{H}}{d \mu^{\prime}} \\
& =\frac{1}{8} A_{d-1}\left(r^{d-1}(M)-r^{d-1}(M-\omega)\right)=\frac{1}{2} \Delta S_{B H}
\end{aligned}
$$

where $\Delta S_{B H}=S_{B H}(M)-S_{B H}(M-\omega)$ is the difference of the entropies of the black hole before and after the emission. Thus, the tunneling probability for the particle is

$$
\Gamma=\exp \left(-\Delta S_{B H}\right) .
$$

If we Taylor expand $\Delta S_{B H}$ in $\omega$, the leading term gives the thermal Boltzmann factors $\exp \left(-\omega / T_{H}\right)$ for the emanating radiation. The second term represents corrections from the response of the background geometry to the emission of a quantum. The same result holds for emission from asymptotically flat black holes [12].

\section{Particle creation by a collapsing spherical shell in AdS}

We will now turn to a third way to analyze Hawking radiation from black holes, and investigate particle creation by a collapsing spherical body which forms a black hole in AdS. We will base our treatment on the discussion in [17], which in turn follows [18] and [19]. As in [17], the starting point is that we assume that in the remote past the spherical body is distended so much that it deforms the anti-de Sitter space. Thus, in the beginning we can assume that a quantum field is in a vacuum constructed with respect to global coordinates in AdS space. Now a convenient choice for the global coordinates is given by

$$
d s^{2}=(\sec \rho)^{2}\left(-d t^{2}+d \rho^{2}\right)+(\tan \rho)^{2} d \Omega_{d-1}^{2} .
$$

The (normalizable) mode solutions can be found e.g. in [20]:

$$
\phi_{n, l}^{(+)}=e^{-i \omega t} Y_{l,\{m\}}(\Omega)(\cos \rho)^{2 h_{+}}(\sin \rho)^{l} P_{n}^{\left(l+\frac{d}{2}-1,2 h_{+}-\frac{d}{2}\right)}(\cos 2 \rho),
$$


where $Y_{l,\{m\}}(\Omega)$ is a spherical harmonic on $S^{d-1}, P_{n}^{\left(l+\frac{d}{2}-1,2 h_{+}-\frac{d}{2}\right)}$ is a Jacobi polynomial, and

$$
2 h_{+}=\frac{d}{2}+\frac{1}{2} \sqrt{d^{2}+4 m^{2}} \equiv \frac{d}{2}+\frac{1}{2} \nu
$$

Due to the boundary conditions at the origin and at the boundary of AdS, the spectrum is discrete, with

$$
\omega=2 h_{+}+2 n+2 l ; n=0,1,2, \ldots
$$

Again, we will focus on $s$-waves $(l=0)$. As in flat space, we expect the quantum to experience a strong redshift as it propagates across the collapsing body. Thus, in the remote past, we are most interested in high frequency modes. In the high frequency limit, the mode solution (46) reduces to a simplified form

$$
\phi_{n}^{(+)} \sim(\cos \rho)^{(d-1) / 2} e^{-i \omega_{n} t} \cos \left(\omega_{n} \rho-\frac{d \pi}{4}\right)
$$

where we suppressed normalization factors. In other words, they take a form of a standing wave, a superposition of an ingoing and outgoing spherical wave, with a discrete spectrum. Note that using the radial coordinate $r$, the overall factor $(\cos \rho)^{(d-1) / 2} \sim(1 / r)^{(d-1) / 2}$ as $r>>1$, so we recover the expected overall decay factor for the amplitude. Now, we will add into the picture the collapsing body and try to compute the redshift due to the passage of the wave across it. As in [17], we will use the geometric optics approximation, and truncate the analysis to two dimensions to the $t, r$-plane by suppressing the overall decay factor of the waveform. For simplicity, we assume that the collapsing body is a thin shell of radius $R$, with $R$ monotonically decreasing in time. The truncated metric inside and outside the shell takes the form

$$
d s^{2}=-F_{ \pm} d t_{ \pm}^{2}+F_{ \pm}^{-1}(r) d r^{2}
$$

where $F_{+}(r)=1-\frac{\mu}{r^{d-2}}+r^{2}$ and $F_{-}(r)=1+r^{2}$. We then define the tortoise coordinates $r_{*}^{ \pm}$,

$$
r_{*}^{ \pm}=\int \frac{d r}{F_{ \pm}(r)}
$$

and the null coordinates

$$
\begin{aligned}
u & =t_{+}-r_{*}^{+}, v=t_{+}+r_{*}^{+} \\
U & =t_{-}-r_{*}^{-}, V=t_{-}+r_{*}^{-}
\end{aligned}
$$

so that the interior and exterior metrics are conformal to a flat metric. The tortoise coordinate in the interior is $r_{*}^{-}=\arctan r$, so the origin $r=0$ corresponds to $r_{*}^{-}=0$. In terms of the null coordinates $U, V$, the origin is then at $V-U=0$. Note also that 
$r_{*}^{-}=\rho$, where $\rho$ is the coordinate that appears in the global metric (45). The exterior and interior null coordinates are related by

$$
\begin{aligned}
v & =\beta(V) \\
U & =\alpha(u)
\end{aligned}
$$

where $\alpha(u)$ and $\beta(V)$ are to be determined below. In the $(t, r)$ coordinates, the passage of a wave across the shell turns to a reflection condition at the origin:

$$
v=\beta(V)=\beta(U)=\beta(\alpha(u)) .
$$

Thus, in the asymptotic region (near the boundary), the waves have a phase structure

$$
\tilde{\phi}^{(+)} \sim e^{-i \omega_{n} v}-e^{-i \omega_{n} \beta(\alpha(u))} .
$$

To find the functions $\alpha, \beta$, we match the interior and exterior metrics across the collapsing shell at $r=R(\tau)$. Here $\tau$ denotes the shell time, which is related to the time coordinates $t_{ \pm}$in the interior and exterior of the shell through

$$
d s^{2}=\left[-F_{ \pm} d t_{ \pm}^{2}+F_{ \pm}^{-1} d r^{2}\right]_{\mid r=R(\tau)}=-d \tau^{2} .
$$

It is easiest to consider the derivatives

$$
\begin{aligned}
& \alpha^{\prime}(u)=\frac{d U}{d u}=\frac{\dot{U}}{\dot{u}} \\
& \beta^{\prime}(V)=\frac{d v}{d V}=\frac{\dot{v}}{\dot{V}},
\end{aligned}
$$

(at the shell) where $\cdot=d / d \tau$. Using the definition (56), we obtain

$$
\begin{aligned}
\frac{d U}{d u} & =\frac{F_{+}(R)\left[\sqrt{F_{-}(R)+\dot{R}^{2}}-\dot{R}\right]}{F_{-}(R)\left[\sqrt{F_{+}(R)+\dot{R}^{2}}-\dot{R}\right]} \\
\frac{d v}{d V} & =\frac{F_{-}(R)\left[\sqrt{F_{+}(R)+\dot{R}^{2}}+\dot{R}\right]}{F_{+}(R)\left[\sqrt{F_{-}(R)+\dot{R}^{2}}+\dot{R}\right]} .
\end{aligned}
$$

As the radius of the shell approaches the horizon, we can approximate

$$
\begin{aligned}
& F_{+}(R) \approx 4 \pi T_{H}\left(R-r_{H}\right) \\
& F_{-}(R) \approx F_{-}\left(r_{H}\right) \equiv A .
\end{aligned}
$$

Then, we can approximate

$$
\frac{d U}{d u} \approx-\frac{2 \pi T_{H}\left(R-r_{H}\right)}{A \dot{R}}\left[\sqrt{A+\dot{R}^{2}}-\dot{R}\right]
$$


where we used $\sqrt{\dot{R}^{2}}=-\dot{R}$ since $\dot{R}<0$ as the shell is collapsing and $|\dot{R}| \neq 0$ as a function of shell time. In the above, $\dot{R}=\dot{R}_{\left.\right|_{r_{H}}}$. Next, we relate $U$ to $R-r_{H}$ by expanding

$$
U \approx U\left(r_{H}\right)+\frac{d U}{d R}_{\mid R=r_{H}}\left(R-r_{H}\right)
$$

and evaluating the derivative $d U / d R$ at the horizon, using the chain rule and the definitions () and (). We obtain

$$
\left(R-r_{H}\right) \approx\left(U-U\left(r_{H}\right)\right) \frac{A \dot{R}}{\left[\sqrt{A+\dot{R}^{2}}-\dot{R}\right]} .
$$

We substitute this to (), and obtain

$$
\frac{d U}{d u} \approx-2 \pi T_{H}\left(U-U\left(r_{H}\right)\right) \equiv-\kappa U+\text { const. }
$$

where $\kappa$ is the surface gravity of the black hole. Integration then gives

$$
\alpha(u)=e^{-\kappa u}+\text { const } .
$$

A similar calculation for $d v / d V$ gives

$$
\left.\frac{d v}{d V} \approx-\frac{A}{\dot{R}\left[\sqrt{A+\dot{R}^{2}}+\dot{R}\right]} \equiv c \quad \text { (= const. }\right)
$$

By integration,

$$
\beta(V)=c V+\text { const }
$$

Thus, we find that in the asymptotic region the waves have a phase structure

$$
\tilde{\phi}^{(+)} \sim e^{-i \omega_{n} v}-e^{-i \omega_{n} c\left(e^{-\kappa u}+\text { const. }\right)} .
$$

To obtain modes where the outgoing wave is of standard form, we invert functionally and write

$$
\tilde{\phi}^{(+)} \sim e^{i \omega_{n} \kappa^{-1} \ln \left[\left(v_{0}-v\right) / c\right]}-e^{-i \omega_{n} u},
$$

which is valid only for $v<v_{0}$. Now, we move back to $(d+1)$ dimensions and compare with the high-frequency limit of the global modes (49). Note that far in the asymptotic region, $r \rightarrow \infty$, the exterior tortoise coordinate reduces to the same form as $\rho, r_{*}^{+} \approx \rho$, so we can write the global mode as

$$
\phi^{(+)} \sim(\cos \rho)^{(d-2) / 2}\left(e^{-i \omega_{n} v-i d \pi / 2}-e^{-i \omega_{n} u}\right) .
$$


We want to compare this with the modes that we found in the collapsing shell geometry,

$$
\tilde{\phi}^{(+)} \sim(\cos \rho)^{(d-2) / 2}\left(e^{i \omega_{n} \kappa^{-1} \ln \left[\left(v_{0}-v\right) / c\right]}-e^{-i \omega_{n} u}\right) \quad\left(v<v_{0}\right)
$$

where we added the overall decay factor. The Bogoliubov transformation follows the discussion in [17], and as a result we find that the outgoing modes are thermally excited, if the field is in a global vacuum. Thus, the global vacuum resembles an Unruh vacuum.

Naturally, the above result only applies to the onset of Hawking radiation as the black hole has formed, and does not address the issue of subsequent evolution. Since the AdS space can be viewed as a finite box, what will happen is that the outgoing radiation cannot escape to infinity but will slowly fill the box. Subsequently, the black hole will come to a thermal equilibrium with the surrounding thermal bath. This situation is descibed by the Hartle-Hawking vacuum in the eternal geometry. Note however that in dimensions $d>2$, if the initial size of the black hole is too small, the equilibrium will not be established before the black hole evaporates completely. See [7] for a thorough analysis.

\section{Note added}

As we were finalizing this paper, the paper [21] appeared, discussing Hawking radiation in the optical collapse geometry for spherically symmetric black holes.

\section{Acknowledgment}

We would like to thank Jorma Louko for useful comments.

\section{References}

[1] J. Maldacena. Adv. Theor. Math. Phys., 2:231, 1998. hep-th/9711200.

[2] O. Aharony, S.S. Gubser, J. Maldacena, H. Ooguri, and Y. Oz. hep-th/9905111.

[3] L. Randall and R. Sundrum. Phys. Rev. Lett., 83:3370, 1999. hep-ph/9905221.

[4] L. Randall and R. Sundrum. Phys. Rev. Lett., 83:4690, 1999. hep-th/9906064.

[5] M. Banados, C. Teitelboim, and J. Zanelli. Phys. Rev. Lett., 69:1849, 1992. hepth/9204099.

[6] S. Hyun, G.H. Lee, and J.H. Yee. Phys. Lett., B322:182, 1994.

[7] G.T. Horowitz and V.E. Hubeny. hep-th/9909056. 
[8] W.G. Unruh. Phys. Rev. D14:870, 1976.

[9] P. Kraus and F. Wilczek. Mod. Phys. Lett. A9:3713, 1994. gr-qc/9406042.

[10] P. Kraus and F. Wilczek. Nucl. Phys. B433:403, 1995. gr-qc/9408003.

[11] M.K. Parikh and Frank Wilczek. hep-th/9907001.

[12] E. Keski-Vakkuri and P. Kraus. Nucl. Phys. B491:249, 1997. hep-th/9610045.

[13] D. Klemm and L. Vanzo. Phys. Rev. D58:104025, 1998. gr-qc/9803061.

[14] E. Keski-Vakkuri. Phys. Rev. D59:104001, 1999. hep-th/9808037.

[15] I. Ichinose and Y. Satoh. Nucl. Phys. B447:340, 1995. hep-th/9412144.

[16] P. Painlevé. C. R. Acad. Sci. (Paris) 173:677, 1921.

[17] N.D. Birrell and P.C.W Davies. Quantum Fields in Curved Space. Cambridge, UK: Univ. Press. (1982).

[18] S.W. Hawking. Comm. Math. Phys. 43:199, 1975.

[19] L. Parker. Phys. Rev. D12:1519, 1975; ibid, in Asymptotic Structure of Space-Time, ed. F.P.Esposito and L. Witten, New York: Plenum Press (1977).

[20] V. Balasubramanian, P. Kraus, and A. Lawrence. Phys. Rev. D59:046003, 1999. hep-th/9805171.

[21] S. Sonego, J. Almergren, and M.A. Abramowicz. gr-qc/0005106. 